\title{
Marc OUIMET
}

CRIMINOLOGUE, ÉCOLE DE CRIMINOLOGIE, UNIVERSITÉ DE MONTRÉAL

(1993)

\section{"L'aigle et le castor : étude de la distribution spatiale de la criminalité aux Etats-Unis et au Canada"}

Un document produit en version numérique par Jean-Marie Tremblay, bénévole, professeur de sociologie au Cégep de Chicoutimi

Courriel: jean-marie tremblay@uqac.ca

Site web pédagogique $:$ http://www.uqac.ca/jmt-sociologue/

Dans le cadre de la collection: "Les classiques des sciences sociales"

Site web: http://classiques.uqac.ca/

Une collection développée en collaboration avec la Bibliothèque

Paul-Émile-Boulet de l'Université du Québec à Chicoutimi

Site web: http://bibliotheque.uqac.ca/ 
Cette édition électronique a été réalisée par Jean-Marie Tremblay, bénévole, professeur de sociologie au Cégep de Chicoutimi à partir de :

\section{Marc OUIMET}

"L'aigle et le castor : étude de la distribution spatiale de la criminalité aux États-Unis et au Canada".

Un article publié dans la revue Criminologie, vol. XXVI, no 2, 1993, pp. 85102. Montréal : École de Criminologie, Université de Montréal.

M Marc Ouimet, criminologue, nous a accordé le 11 septembre 2006 son autorisation de diffuser électroniquement cet article.

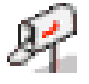

Courriel : marc.ouimet@umontreal.ca ou ouimetm@ere.umontreal.ca

Polices de caractères utilisée :

Pour le texte: Times New Roman, 14 points.

Pour les citations : Times New Roman, 12 points.

Pour les notes de bas de page : Times New Roman, 12 points.

Édition électronique réalisée avec le traitement de textes Microsoft Word 2004 pour Macintosh.

Mise en page sur papier format : LETTRE (US letter), 8.5” x 11”)

Édition numérique réalisée le 23 novembre 2006 à Chicoutimi, Ville de Saguenay, province de Québec, Canada.

\section{Fait avec}




\section{Table des matières}

Résumé

Introduction

1. LES PRINCIPAUX MODELES D'EXPLICATION DES DIFFERENCES DE LA CRIMINALITÉ ENTRE LES ÉTATS-UNIS ET LE CANADA

2. UN PREMIER REGARD : LES DIFFÉRENCES ENTRE LES ÉTATSUNIS ET LE CANADA

3. UN SECOND REGARD : LES DIFFÉRENCES ENTRE DEUX PAYS COMPARABLES

\section{4. $\quad$ LA DISTRIBUTION SPATIALE DES TAUX DE CRIMINALITÉ}

Tableau 1. Le taux de criminalité aux ÉtatsUnis et au Canada en 1990

Tableau 2. La composition des deux pays « comparables » en 1990

Tableau 3. Le taux de criminalité pour les ÉtatsUnis du Nord et le Canada en 1990

Tableau 4. Corrélations des taux de criminalité entre les états américains et les provinces canadiennes $(\mathrm{n} .=59)$

Carte 1. La répartition spatiale des homicides

Carte 2. La répartition spatiale des vols qualifiés

Carte 3. La répartition spatiale des vols par effraction

Carte 4. La répartition spatiale des vols de véhicules à moteur

Conclusion

Bibliographie 
Marc OUIMET ${ }^{1}$

"L'aigle et le castor: étude de la distribution spatiale de la criminalité aux États-Unis et au Canada”. 2

Un article publié dans la revue Criminologie, vol. XXVI, no 2, 1993, pp. 85102. Montréal: École de Criminologie, Université de Montréal.

\section{Résumé}

\section{$\underline{\text { Retour à la table des matières }}$}

In the fast three decades, a number of researchers have undertook the comparison of American and Canadian crime rates. Among them, Lipset (1990) and Hagan (1991) have shown that violence was more frequent south of the border than north of it. To explain why crime was more frequent in the US than in Canada, those authors argued that differences in values and culture of each country's residents was the principal determinant of this situation. Using regional and infranational disaggregated crime rates, this article shows that differences in both country's crime rates are not univocal. For example, crime rates in Canada are not higher than those of Northern United States for three crimes out of four studied. What makes US crime rates appear much higher than the Canadian ones can be attributable to a small number of States and cities which have extraordinarily high crime rates.

1 L'auteur est professeur à l'École de criminologie, Université de Montréal, C.P. 6128, Succ. A », Montréal, Québec, H3C 317.

2 II convient de remercier le fonds FCAR, pour l'apport financier à ce projet de recherche, et Jean Paiement, pour l'aide apportée à la réalisation de cette étude. Le document inédit Le meurtre aux ÉtatsUnis et au Canada : Essai de criminologie comparée de Maurice Cusson a servi de guide à notre réflexion. 


\section{INTRODUCTION}

$\underline{\text { Retour à la table des matières }}$

$\mathrm{Au}$ cours des trois dernières décennies, bon nombre de travaux de recherche ont comparé les taux de criminalité entre les États-Unis et le Canada. La plupart des études disponibles visent d'abord à expliquer des différences apparentes entre les taux d'homicides des deux pays. Cette approche limitée du problème comporte deux faiblesses. En premier lieu, l'homicide n'est pas nécessairement représentatif de l'ensemble de la criminalité. En second lieu, l'empressement à expliquer les différences observables n'a pas permis une analyse détaillée des différences etiou des ressemblances entre les deux pays. La présente étude, exploratoire, vise donc à étayer les connaissances comparatives sur la criminalité aux États-Unis et au Canada. Son objectif est de circonscrire les différences et les ressemblances de manière à orienter les modèles explicatifs qui peuvent en rendre compte.

L'étude des comparaisons internationales a une longue histoire en criminologie. Cependant, ce type d'analyse est confronté à de nombreux problèmes, notamment à ceux liés à la disponibilité et à la validité des données empiriques. Killias (1991) présente les principaux dangers liés aux comparaisons internationales reposant sur les indicateurs officiels de la criminalité. Il préconise une approche de comparaison en profondeur, entre deux ou trois pays à la fois. Avant d'entreprendre une comparaison internationale, il faut pouvoir compter sur des indicateurs statistiques comparables. Les crimes d'homicide, de vol qualifié, de vol par effraction et de vol de véhicule à moteur sont les plus souvent utilisés à des fins de comparaison. Ces crimes ont l'avantage principal d'être souvent rapportés à la police par leurs victimes (Cusson, 1990).

Le chiffre noir de la criminalité ne constitue pas un problème pour l'analyse comparative dans le cas où le taux de reportabilité est constant à travers les différents territoires considérés. Même si des données 
précises à cet égard ne sont actuellement disponibles qu'à l'état parcellaire, il n'y a pas a priori de raison de croire que les taux de reportabilité soient très différents entre le Canada et les ÉtatsUnis, d'autant que les taux de reportabilité entre les différents états américains varient peu (Hindelang et al., 1981). Cette question mériterait toutefois d'être abordée plus à fond, en particulier à partir du sondage canadien de victimisation de 1993. En ce qui a trait à la définition légale et opérationnelle des quatre crimes mentionnés, il n'y a pas de différence majeure entre les définitions adoptées aux ÉtatsUnis et au Canada qui puisse expliquer la plus ou moins grande prévalence dans l'un d'eux.

\section{LES PRINCIPAUX MODÈLES D'EXPLICATION DES DIFFERENCES DE LA CRIMINALITÉ ENTRE LES ÉTATS-UNIS ET LE CANADA}

$\underline{\text { Retour à la table des matières }}$

Les premiers travaux de criminologie comparée poursuivis aux États-Unis et au Canada montrèrent que la criminalité était plus importante au sud de la frontière qu'au nord de celleci (Clark, 1976). Lipset (1990) montre bien les différences qui ont marqué la colonisation de la partie ouest des deux pays. Aux ÉtatsUnis, les colons se sont d'abord implantés et les corps policiers se sont ensuite organisés. Au Canada, la Gendarmerie Royale s'est installée avant l'arrivée des colons. À cette époque, les Américains étaient populistes et se montraient méfiants à l'égard du pouvoir central. Au contraire, les Canadiens défendaient un idéal anti-révolutionnaire et respectaient les traditions britanniques. Bien que ces divergences historiques soient réelles, il serait toutefois inopportun de leur accorder une trop grande importance dans l'explication d'éventuelles différences observables en 1990.

Le politicologue Seymour Martin Lipset a analysé les écarts existants entre les taux de criminalité des États-Unis et du Canada, dans le cadre d'une étude plus générale des sociétés canadienne et américaine. Dans Continental Divide: The values and institutions in the United 
States and Canada (1990), Lipset tente de cerner les facteurs expliquant pourquoi l'homicide, les protestations politiques et la consommation de drogues illicites sont plus répandus au sud de la frontière qu'au nord de celle-ci. Lipset indique que la plus grande prévalence de la violence et de la déviance aux ÉtatsUnis est due au fait que les Américains sont plus individualistes que les Canadiens, et qu'ils voient d'un mauvais oeil l'intervention de l'État dans leur vie privée. Par comparaison, les Canadiens sont davantage prêts à sacrifier une part de leur liberté pour le bien de la majorité. Ils sont plus souvent favorables à restreindre le port d'armes à feu, l'usage du tabac dans des lieux publics et la circulation routière dans les villes. De plus, les Canadiens portent un jugement plus favorable sur la police que les Américains. Lipset croit cependant que la création de la Charte des droits et libertés au Canada, par l'entremise de son impact sur les pratiques policières et judiciaires, va entraîner le Canada vers une américanisation plus rapide que ne peut le faire le traité de libre-échange nordaméricain.

Lipset (1990) met donc de l'avant une interprétation culturelle des différences entre les États-Unis et te Canada. Or, si les citoyens des deux pays se distinguent sur le plan des valeurs et que cellesci ont un rapport quelconque avec la criminalité, il y a lieu de se demander pourquoi les écarts des taux de criminalité varient considérablement d'une conduite criminelle à l'autre. Le criminologue torontois John Hagan apporte certains éléments de réponse à cette question. Dans Disreputable Pleasures : Crimes and deviance in Canada (1991), il analyse un éventail de données relatives au crime dans les deux pays, y compris des données policières, des sondages de victimisation et des données issues des enquêtes de délinquance autorévélées. Hagan indique que plus une activité criminelle est grave, plus la différence entre le Canada et les États-Unis est importante. L'accessibilité des armes à feu aux États-Unis peut donc expliquer pourquoi les crimes violents y sont si nombreux. De manière plus générale, Hagan note que les Américains et les Canadiens n'ont pas instauré de semblables stratégies de contrôle social du crime. Les Américains tolèrent plus facilement la déviance, en particulier celle des personnes défavorisées, mais interviennent avec force pour arrêter et incarcérer les individus qui ont dépassé les limites de l'acceptable. Pour leur part, les Canadiens sont plus sensibles aux problèmes d'adaptation sociale de leurs concitoyens 
et, parce qu'ils attachent beaucoup d'importance à l'ordre social, découragent toute déviance aux normes. Hagan, qui explique les différences entre les deux pays en termes de valeurs et de culture, s'inscrit donc directement dans la voie tracée auparavant par Lipset.

Les explications des différences de criminalité entre les États-Unis et le Canada peuvent s'inscrire aussi dans d'autres cadres conceptuels. Selon un modèle structuraliste, le taux de criminalité élevé des ÉtatsUnis s'explique par une plus grande importance des inégalités sociales et de la discrimination raciale dans ce pays (Lenton, 1989). Bien sûr, l'écart entre les valeurs partagées par les citoyens des deux pays a nécessairement un lien avec la discrimination et les inégalités sociales, mais il n'est qu'indirect et diffus. Lenton montre que le taux d'homicide des Noirs américains et des hispanophones est si élevé qu'il explique près de la moitié de la différence constatée avec le Canada. La chose est également vraie au Canada, où les autochtones, qui ne représentent qu'environ $2 \%$ de la population, comptaient pour $21 \%$ des homicides en 1986. Des analyses dérivées montrent que le taux d'homicide des Américains blancs n'est que de 1,8 fois supé rieur au taux des Canadiens blancs, ce qui est nettement moins important que le ratio de 3,9 calculé par Hagan (1990). Selon Lenton, qui s'inscrit dans le courant de structuralistes tels que Horrowitz (1973), les personnes issues des minorités ethniques sont surreprésentées dans les prisons, parce que la présence de la violence à l'intérieur de leur communauté est plus grande que celle observée à l'intérieur de la majorité blanche. La discrimination dont sont victimes les personnes issues des minorités ethniques et la pauvreté qui en résulte ont un effet indirect sur l'homicide, en raison principalement de la désorganisation familiale, et un effet direct sur l'homicide. Or, dès 1971, Truman indiqua que les États-Unis se distinguaient des autres démocraties d'origine britannique (i.e. l'Australie, les États-Unis et le Canada) par l'existence d'importants ghettos noirs.

L'examen de la documentation sur la question montre clairement que l'explication des différences observées entre les taux d'homicide des deux pays fut la principale préoccupation des auteurs ayant abordé le sujet. De plus, aucune étude n'a analysé d'éventuelles différences de fréquence et de prévalence de la criminalité entre les deux pays. Selon une conceptualisation dérivée des travaux de Blumstein et al. (1986), 
un écart des taux de criminalité peut s'expliquer par une plus grande prévalence de ce comporte ment dans un endroit plutôt que dans un autre (i.e. qu'un plus fort pourcentage de la population prend part au crime), par une plus grande fréquence individuelle de ce comportement criminel (i.e. que le nombre annuel de crimes commis par un délinquant est plus élevé), ou par l'effet combiné de ces deux facteurs. Ce type d'interrogation a des chances d'être fructueux, car les facteurs influençant la prévalence (i.e. pauvreté, familles brisées, etc.) ne sont pas nécessairement les mêmes que ceux expliquant la fréquence (Le. opportunités, impunité, etc.). Malheureusement, cet axe d'interprétation ne peut être exploré plus à fond pour le moment, étant donné l'absence de données utiles à ce genre d'entreprise ${ }^{3}$.

Mis à part une trop grande attention accordée à l'homicide et l'absence d'une conceptualisation qui distingue entre fréquence et prévalence, la majorité des travaux comparatifs existants n'ont pas considéré le problème du niveau d'agrégation spatiale des données statistiques ${ }^{4}$. Est-ce que des citoyens vivant dans des zones géographiques comparables ont des probabilités de victimisation différentes ? Les données de Sloan et al. (1988) montrent que le taux de victimisation par homicide des résidents blancs de Vancouver est de 6,2 par 100000 habitants alors qu'il est de 6,4 pour les Blancs de Seattle. Il se peut donc que le banlieusard de Boston ait les mêmes probabilités d'être victime que le banlieusard de Montréal ! Par conséquent, il est possible que les différences entre le taux de criminalité des deux pays s'expliquent tout simplement par des répartitions inégales des citoyens dans des zones à plus ou moins grande densité de criminalité. Le taux de criminalité plus élevé aux ÉtatsUnis peut s'expliquer par le fait qu'il existe un plus grand nombre de zones à hauts risques, comme par exemple le sud du pays et les territoires métropolitains de quelques grandes villes caractérisées par la présence de ghettos. Cette vision du problème a comme caractéristique principale de rejeter l'interprétation

3 Il faudrait mener un sondage de délinquance révélée dans la population pour estimer la prévalence et un sondage de délinquance révélée chez les prisonniers pour estimer la fréquence.

4 Voir Élie et Cusson (1992) pour une analyse approfondie du problème d'agrégation spatiale des données statistiques. 
culturelle directe de l'explication des différences observables des taux de criminalité entre les deux pays.

L'hypothèse de travail de la présente étude est donc que la probabilité de victimisation d'individus comparables, vivant dans des villes et des territoires comparables, est équivalente pour le Canadien et l'Américain. En utilisant les états et les provinces comme unités d'analyse, il est possible de vérifier si des territoires ayant des caractéristiques semblables ont des taux de criminalité comparables. Cette hypothèse de recherche est dérivée du constat effectué par Lenton (1989) qui montre que la variabilité des taux de criminalité est plus grande à l'intérieur des États-Unis et du Canada qu'entre ces deux pays.

\section{UN PREMIER REGARD : LES DIFFÉRENCES ENTRE LES ÉTATS-UNIS ET LE CANADA}

$\underline{\text { Retour à la table des matières }}$

Cette section présente les taux de criminalité entre les ÉtatsUnis et du Canada pris dans leur ensemble. C'est à ce niveau d'agrégation que se sont situés la plupart des auteurs ayant traité des différences entre les deux pays. Les données statistiques canadiennes proviennent du catalogue annuel de Statistique Canada portant sur la criminalité au Canada (1991). Les données américaines lurent puisées dans le Sourcebook of Criminal Justice Statistics (1991) publié annuellement par le U.S. Department of Justice. Le tableau 1 rapporte les taux de criminalité et le ratio entre les ÉtatsUnis et le Canada pour l'année 1990 5 . Les données canadiennes n'incluent pas le Yukon et les Territoires du Nord-Ouest, alors que les données américaines excluent cel-

5 Aucune statistique de signification n'est présentée, parce que les données ne sont pas issues d'échantillons représentatifs de la population des territoires considérés. Les taux utilisés peuvent donc être considérés comme des paramètres. 
les d'Hawaï et de Puerto Rico ${ }^{6}$. Un ratio négatif indique que le taux de criminalité est plus élevé au Canada qu'aux États-Unis.

Tableau I

Le taux de criminalité aux États-Unis et au Canada en 1990

$\underline{\text { Retour à la table des matières }}$

\begin{tabular}{lllll}
\hline & Homicide & $\begin{array}{c}\text { Vol quali- } \\
\text { fié }\end{array}$ & $\begin{array}{l}\text { Vol par ef- } \\
\text { fraction }\end{array}$ & $\begin{array}{l}\text { Vol de véhi- } \\
\text { cule moteur }\end{array}$ \\
\hline États-unis & 9,44 & 2,58 & 12,61 & 6,59 \\
Canada & 2,43 & 1,06 & 14,19 & 4,26 \\
Ratio É.-U.-CAN & 3,89 & 2,43 & $-1,13$ & 1,55 \\
\hline
\end{tabular}

Le taux d'homicide aux ÉtatsUnis est très élevé et figure parmi les plus hauts des pays industrialisés. Selon Gantier (1990), si les ÉtatsUnis forment le pays où l'homicide est le plus fréquent parmi dix-huit démocraties occidentales, le Canada se classe troisième. Le taux d'homicide au Canada est plus élevé que celui observé, par exemple, en France, en Italic et en Belgique. De façon générale, on peut dire que le ratio entre le Canada et les États-Unis se situe entre 3 et 4, ce qui représente une différence extrêmement importante entre les deux pays.

En ce qui a trait au vol qualifié, les Américains doivent faire face 2,4 fois plus souvent à ce genre de crimes que les Canadiens. Il est difficile de savoir si la structure de ces vols qualifiés est similaire dans les deux pays. Il est possible qu'une partie de la différence entre les deux pays s'explique par une plus grande prévalence $d u$ mugging (i.e. un vol qualifié dans la rue) aux ÉtatsUnis qu'au Canada. En effet, le mugging est moins grave que le vol qualifié d'un commerce, mais il

6 Ces territoires présentent des caractéristiques trop différentes des autres territoires des deux pays pour être considérés. Leur faible importance démographique ne peut affecter l'économie générale des résultats. 
est aussi moins payant, ce qui force le délinquant à répéter son geste. Les données comparatives sur les différents types de vols qualifiés sont toutefois insuffisantes pour permettre de répondre à cette dernière question.

Le tableau I indique que le vol de véhicule à moteur est plus fréquent chez les Américains que chez les Canadiens ; le ratio entre les deux pays est de 1,6. D'autre part, le Canada connaît plus de vols par effraction que les ÉtatsUnis observation qui peut paraître surprenante à première vue. Ainsi, pour ce qui est des crimes de gravité moyenne, il semble que les deux pays soient à première vue relativement homogènes.

Les différences canadoaméricaines dans la prévalence des crimes contre la propriété sont bien moins grandes que celles observées dans le cas de l'homicide. Cette observation suggère donc que l'homicide ne répond pas aux mêmes lois que des crimes comme le vol qualifié ou le vol par effraction. Dans le cadre d'une comparaison entre les ÉtatsUnis et le Canada, l'homicide apparaît donc comme un cas particulier. Comparer la criminalité entre les deux pays en n'utilisant que l'homicide fournit donc une image partielle et biaisée de la réalité. Les motivations propres à l'homicide (i.e. la vengeance, la colère, etc.) doivent donc être comparées à celles des crimes contre la propriété. Les théories culturelles développées par Lipset (1990) et Hagan (1991) n'apportent pas d'explication à ce sujet.

La comparaison des données agrégées à l'ensemble des deux pays pose un problème de validité. Comme l'a mentionné Lenton (1989), les variations intranationales des taux de criminalité sont plus importantes que les variations internationales. Il serait donc faux de croire que le taux d'homicide se rapproche dc 9,4 pour la majorité des états américains et qu'il se rapproche de 2,4 pour la majorité des provinces canadiennes. Certains états américains ont des taux d'homicides inférieurs à 2 par 100000 (par exemple, l'Iowa et le New Hampshire), alors que certaines provinces canadiennes ont des taux d'homicide supérieurs à 3 par 100000 habitants (par exemple, le Manitoba et le Colombie-Britannique). On ne peut ignorer cette critique qui constitue le fondement de la stratégie analytique de cette étude. 
De manière à circonscrire les facteurs pouvant expliquer une différence observée entre deux territoires, il est préférable de se référer à des unités de comparaison les plus semblables possible. Par exemple, il serait plus utile à notre propos de comparer le Québec et l'Ontario que de comparer le Québec et le NouveauBrunswick. De la même manière, il serait profitable de comparer le Canada et les États-Unis du Nord pour connaître les différences réelles existant entre ces deux pays.

\section{UN SECOND REGARD : LES DIFFÉRENCES ENTRE DEUX PAYS COMPARABLES}

$\underline{\text { Retour à la table des matières }}$

Brantingbam et Brantingham (1984) ont procédé à une comparaison du taux de criminalité entre divers pays. Par contre, ces auteurs préconisent plutôt l'utilisation des comparaisons régionales de manière à mieux isoler les différences réelles existant entre les états. Pour obtenir deux pays comparables, il serait utile de coupler chacune des provinces canadiennes à un état américain semblable, et de comparer ainsi le taux de criminalité de ces deux pays. S'il est possible de comparer le Québec et le Massachusetts ces territoires font partie du Nordest, et Boston et Montréal se ressemblent à plusieurs égards -, la province de l'Ontario ne trouve pas de jumelle américaine, puisque Toronto ne ressemble ni à Detroit ni à Chicago.

S'il est impossible de coupler systématiquement les provinces et les états, il est néanmoins permis de recréer un pays appelé États-Unis et qui ressemblerait au Canada. Les états américains situés à proximité de la frontière canadienne furent choisis en premier pour faire partie des États-Unis du Nord. La population des états fut aussi utile à l'élaboration d'un pays comparable au Canada. Le choix définitif des états fut effectué sur la base d'une méthode intuitive, qui nous parut moins mécanique que celle hautement empirique reposant sur l'analyse des typologies (i.e. cluster analysis). Il est important de noter que les décisions relatives à la composition des États-Unis du Nord furent prises sans égard aux taux de criminalité des différents territoires. Le tableau 
2 présente les informations relatives à la composition des deux pays comparables.

\section{Tableau 2}

La composition des deux pays « comparables » en 1990

$\underline{\text { Retour à la table des matières }}$

\begin{tabular}{lcl}
\hline & Canada & \\
\hline Province & & Population \\
Île du Prince Édouard & 530300 \\
Terre Neuve & 574400 \\
Nouvelle-Écosse & 894200 \\
Nouveau-Brunswick & 723200 \\
Québec & 6767000 \\
Ontario & 9743300 \\
Manitoba & 1091600 \\
Saskatchewan & 999500 \\
Alberta & 2471600 \\
Colombie-Britannique & 3126600 \\
Population totale & 26523700 \\
& États-Unis \\
État & \\
Maine & 1227900 \\
New Hampshire & 1109300 \\
Vermont & 562800 \\
Massachusetts & 6016400 \\
Wisconsin & 4891800 \\
Minnesota & 4375100 \\
North Dakota & 638800 \\
South Dakota & 696000 \\
Montana & 799000 \\
Washington & 4866600 \\
Population totale & 25183800 \\
\hline
\end{tabular}


Lorsque les dix états américains sélectionnés et les dix provinces canadiennes sont considérés, les ÉtatsUnis du Nord comptent 25.2 millions d'habitants et le Canada compte 26,5 millions d'habitants pour l'année 1990. Il serait possible de débattre longtemps de la valeur de cette composition des États-Unis du Nord. Des analyses de la composition des populations (par exemple, le pourcentage de citoyens vivant en milieu urbain, l'incidence de la pauvreté, etc.) pourraient être effectuées pour valider cette composition. Il est de notre avis que cette composition a une validité de surface (i.e. face validity) et que le potentiel heuristique des analyses effectuées avec des pays comparables en justifie l'utilisation. Le tableau 3 présente les taux de criminalité par 100000 habitants pour le Canada et pour les ÉtatsUnis du Nord.

Tableau 3

Le taux de criminalité pour les États-Unis du Nord et le Canada en 1990

$\underline{\text { Retour à la table des matières }}$

\begin{tabular}{lllll}
\hline & Homicide & $\begin{array}{c}\text { Vol } \\
\text { qualifié }\end{array}$ & $\begin{array}{c}\text { Vol par } \\
\text { effraction }\end{array}$ & $\begin{array}{c}\text { Vol de véhicule à } \\
\text { moteur }\end{array}$ \\
\hline États-Unis & 3,75 & 1,19 & 9,58 & 4,90 \\
Canada & 2,43 & 1,06 & 14,19 & 4,26 \\
Ratio EU-CAN & 1,54 & 1,12 & $-1,48$ & 1,15 \\
\hline
\end{tabular}

Lorsque les taux de criminalité du Canada sont comparés à ceux des ÉtatsUnis du Nord, il appert que les différences antérieurement observées s'estompent considérablement Le taux d'homicide des ÉtatsUnis du Nord n'est plus qu'une fois et demie supérieur à celui du Canada, alors que les taux pour le vol qualifié et le vol de véhicule à moteur se ressemblent des deux côtés de la frontière. Toutefois, la différence originale du taux de vol par effraction entre le Canada et les ÉtatsUnis s'est amplifiée (de $1,13: 1$ à $1,48: 1$ ).

Compte tenu du fait que le taux de vol de véhicule à moteur et le taux de vol qualifié sont comparables entre les deux pays, il est possible d'en conclure que la criminalité n'est pas plus élevée au sud de la 
frontière qu'au nord de celleci, lorsque des territoires semblables sont comparés. Les homicides demeurent plus fréquents, ce qui peut s'expliquer par une plus grande disponibilité des armes à feu aux EtatsUnis ${ }^{7}$. D'ailleurs, cette plus grande disponibilité des armes à feu en sol américain peut expliquer pourquoi le vol par effraction y est moins répandu : les risques de sanction immédiate (i.e. être tué par le propriétaire) sont plus grands aux États-Unis qu'au Canada (Kleck, 1979) ${ }^{8}$. Ces résultats tendent donc à montrer que, de façon générale, les Canadiens et Américains vivant dans des états et des provinces comparables sur le plan géographique et démographique ne se distinguent pas les uns les autres sur le plan de leur probabilité respective de victimisation.

\section{LA DISTRIBUTION SPATIALE DES TAUX DE CRIMINALITÉ}

$\underline{\text { Retour à la table des matières }}$

De manière à apprécier plus intuitivement les différences entre les ÉtatsUnis et le Canada, il convient d'illustrer la répartition spatiale du taux de criminalité sur le territoire couvert par les deux pays à l'aide de cartes thématiques. Par contre, avant de procéder à une analyse semblable, il importe de connaître la constante des étais et des provinces sur le plan de la fréquence relative des quatre conduites étudiées. En d'autres mots, y a-t-il une constante dans la prévalence des homicides, des vols qualifiés, des vols par effraction et des vols de véhicules à moteur à l'intérieur des cinquante-neuf territoires étudiés ? Le tableau 4 indique les corrélations entre les taux de criminalité pour les provinces et les états du continent.

7 Wright et al. (1982) estiment qu'il y avait entre 100 millions et 140 millions d'armes à feu aux ÉtatsUnis vers la fin des années soixantedix. Environ un tiers de ces armes sont des revolvers.

8 Kleck (1979) estima que les risques d'appréhension et de condamnation parle système judiciaire pour un vol par effraction étaient d'environ $2 \%$, ce qui est comparable à la probabilité qu'un voleur soit tiré à bout portant (i.e. being shot) par le propriétaire d'une maison occupée. 
Tableau 4

Corrélations des taux de criminalité entre les états américains et les provinces canadiennes $(n .=59)$

$\underline{\text { Retour à la table des matières }}$

\begin{tabular}{lllll}
\hline & Homicide & $\begin{array}{c}\text { Vol } \\
\text { qualifié }\end{array}$ & $\begin{array}{c}\text { Vol par } \\
\text { effraction }\end{array}$ & $\begin{array}{c}\text { Vol de véhicule à } \\
\text { moteur }\end{array}$ \\
\hline Homicide & 0,71 & 0,38 & 0,56 & 0,55 \\
Vol qualifié & - & 0,36 & 0,82 & 0,63 \\
Vol par effraction & - & - & 0,44 & 0,39 \\
Vol de véhicule à & - & - & - & 0,61 \\
moteur & & & &
\end{tabular}

Selon le tableau 4, il appert que les corrélations observables entre les taux de criminalité pour les quatre formes de criminalité étudiées sont modérées. En effet, une corrélation moyenne globale de 54,5 entre les quatre types de taux de criminalité ne peut être considérée comme élevée. D'ailleurs, les corrélations présentées surestiment la force de la relation, parce qu'elles incluent des étais se distinguant par des taux de criminalité très élevés sur l'ensemble des quatre types de crimes. Toute théorie visant à expliquer les différences de taux de criminalité sur le plan macrosociologique doit être en mesure d'expliquer pourquoi un type donné d'activité criminelle est fréquent à un endroit en particulier, alors que d'autres types de crimes ne sont pas particulièrement fréquents au même endroit L'inconstance observée entre les taux de criminalité des états et des provinces complique nécessairement le développement d'une macro-théorie explicative de la répartition spatiale de la criminalité.

De manière à permettre la visualisation des concentrations spatiales de l'activité criminelle au niveau du territoire couvert par les ÉtatsUnis et le Canada, quatre cartes thématiques furent constituées. La procédure suivie pour la mise au point de cartes thématiques fut la suivante. D'abord une distribution de fréquence pour chacun des types d'activité criminelle fut effectuée sur les cinquanteneuf territoires étu- 
diés. Ensuite, les distributions de fréquence furent recodées en trois groupes de même importance. Les cartes 1 à 4 représentent donc la concentration d'activité criminelle en trois degrés d'intensité pour les états américains et les provinces canadiennes. Les territoires blancs indiquent une faible activité criminelle, les territoires gris indiquent une activité criminelle moyenne, alors que les territoires noirs indiquent une prévalence importante du type de criminalité en question.

La carte 1 illustrant la répartition spatiale de l'homicide est sans contredit celle qui a les concentrations les plus systématiques. Une nette diagonale sud/sudouest est observable. C'est à partir de cette distribution spatiale de l'homicide que le concept de southerness fut développé (Gastil, 1971). Selon Gastil, les habitants du Sud se distinguent des autres Américains par leur appartenance à une sousculture de violence répandue dans des états comme la Louisiane ou le Texas ${ }^{9}$. À partir d'une enquête téléphonique, Wright et al. (1982) démontrent que $72 \%$ des résidents du sud des ÉtatsUnis affirment posséder une arme à feu à la maison, contre seulement $24 \%$ des répondants de la NouvelleAngleterre. La carte de la distribution spatiale de l'homicide montre aussi que toutes les provinces canadiennes sont classées parmi les territoires à faible prévalence. Même les provinces canadiennes où l'homicide est le plus fréquent (le Manitoba : 3,57; la Saskatchewan : 3,60 ; la Colombie Britannique : 3,52) ne peuvent se qualifier dans le groupe des territoires à taux d'homicide moyennement élevé. Au Canada, on a remarqué que les taux d'homicide variaient, par ordre croissant, d'est en ouest (Kennedy et al.. 1988). Les taux d'homicide élevés des provinces de l'Ouest s'expliquent en partie par les taux d'homicides au sein de la communauté autochtone. Silverman et Kennedy (1993) indiquent que le taux d'homicide des autochtones canadiens, qui habitent en très grande majorité dans les Prairies, est de l'ordre de 25 par 100000 habitants, soit un taux dix fois plus élevé que celui observé dans l'ensemble des Canadiens.

9 La thèse de l'existence d'une sousculture de violence dans le sud des ÉtatsUnis fut l'objet de maintes recherches. Lizotte et Bordua (1980) remettent en question la validité de ce concept et y opposent des facteurs comme la pauvreté, la ruralité et l'intérêt pour les armes à feu et la chasse. 


\section{Carte 1}

La répartition spatiale des homicides

$\underline{\text { Retour à la table des matières }}$

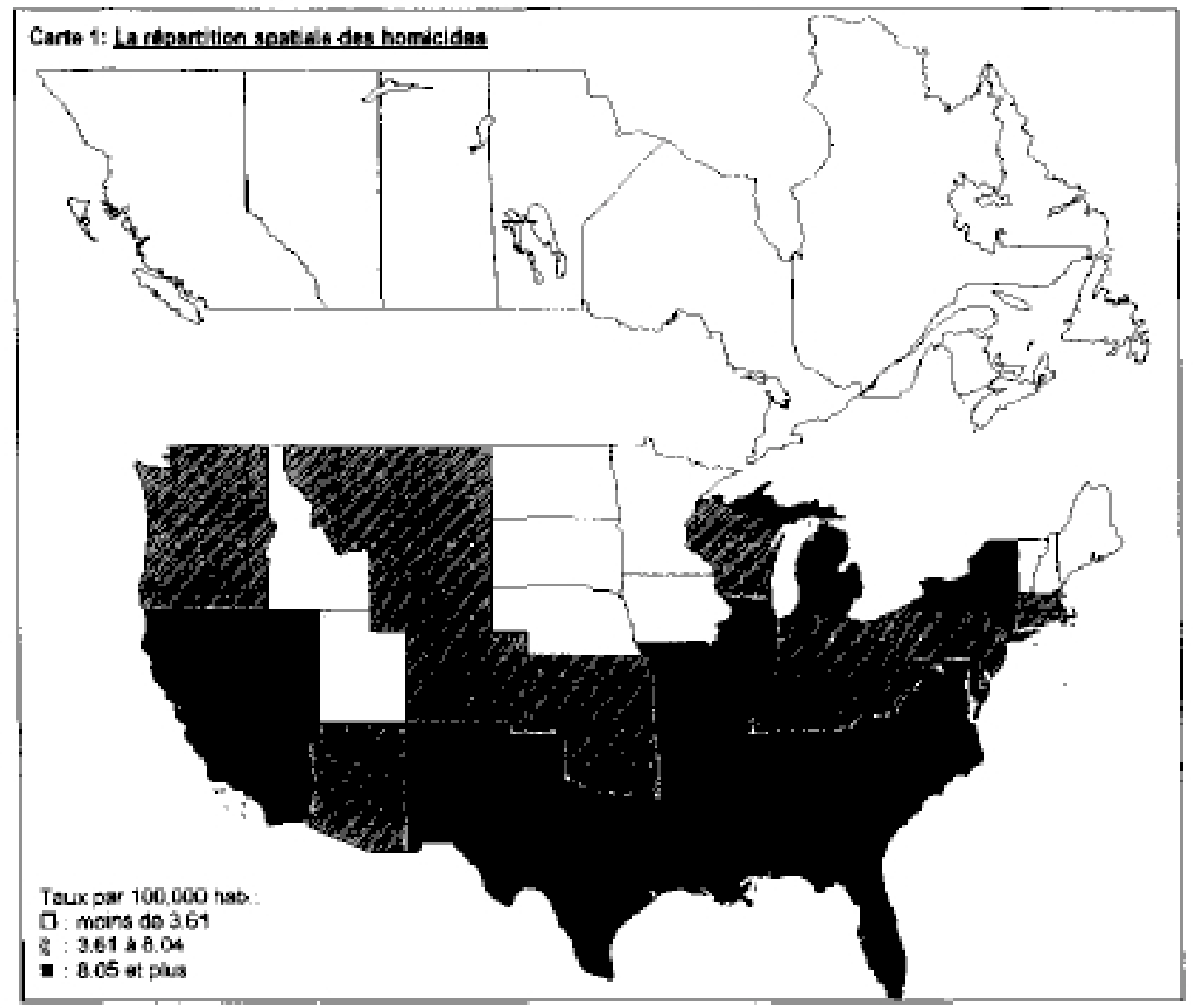




\section{Carte 2}

La répartition spatiale des vols qualifiés

$\underline{\text { Retour à la table des matières }}$

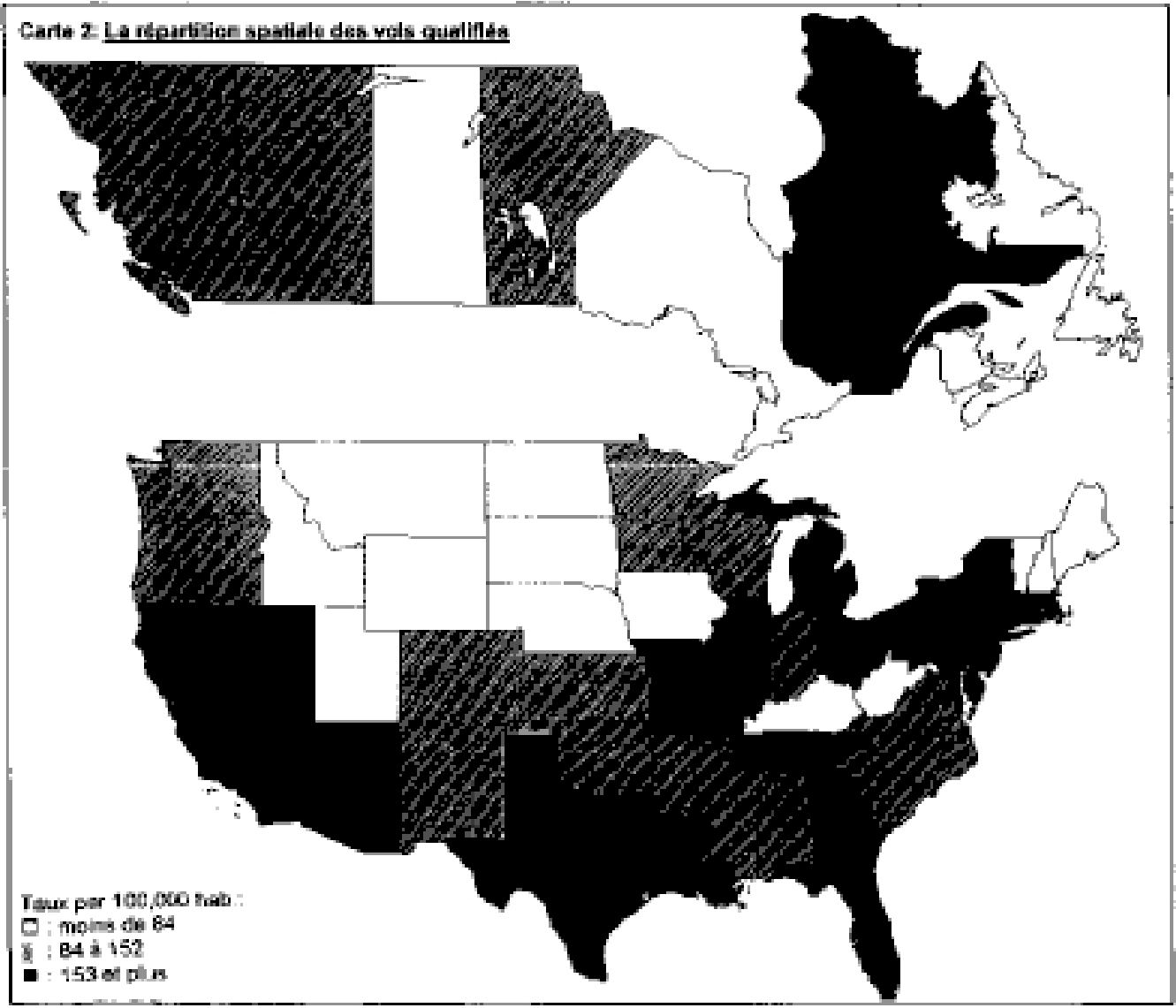




\section{Carte 3}

La répartition spatiale des vols par effraction

\section{$\underline{\text { Retour à la table des matières }}$}

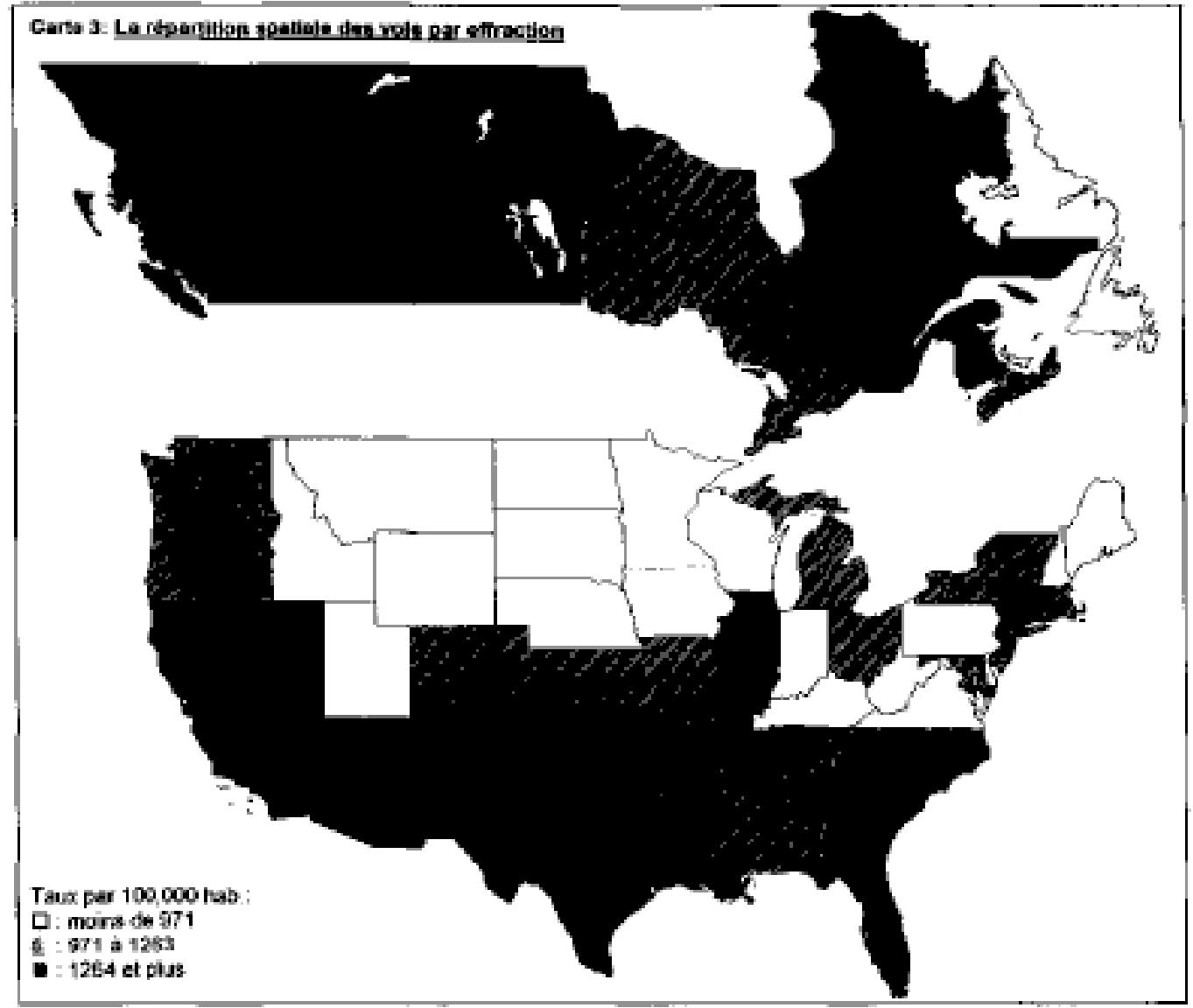




\section{Carte 4}

\section{La répartition spatiale des vols de véhicules à moteur}

$\underline{\text { Retour à la table des matières }}$

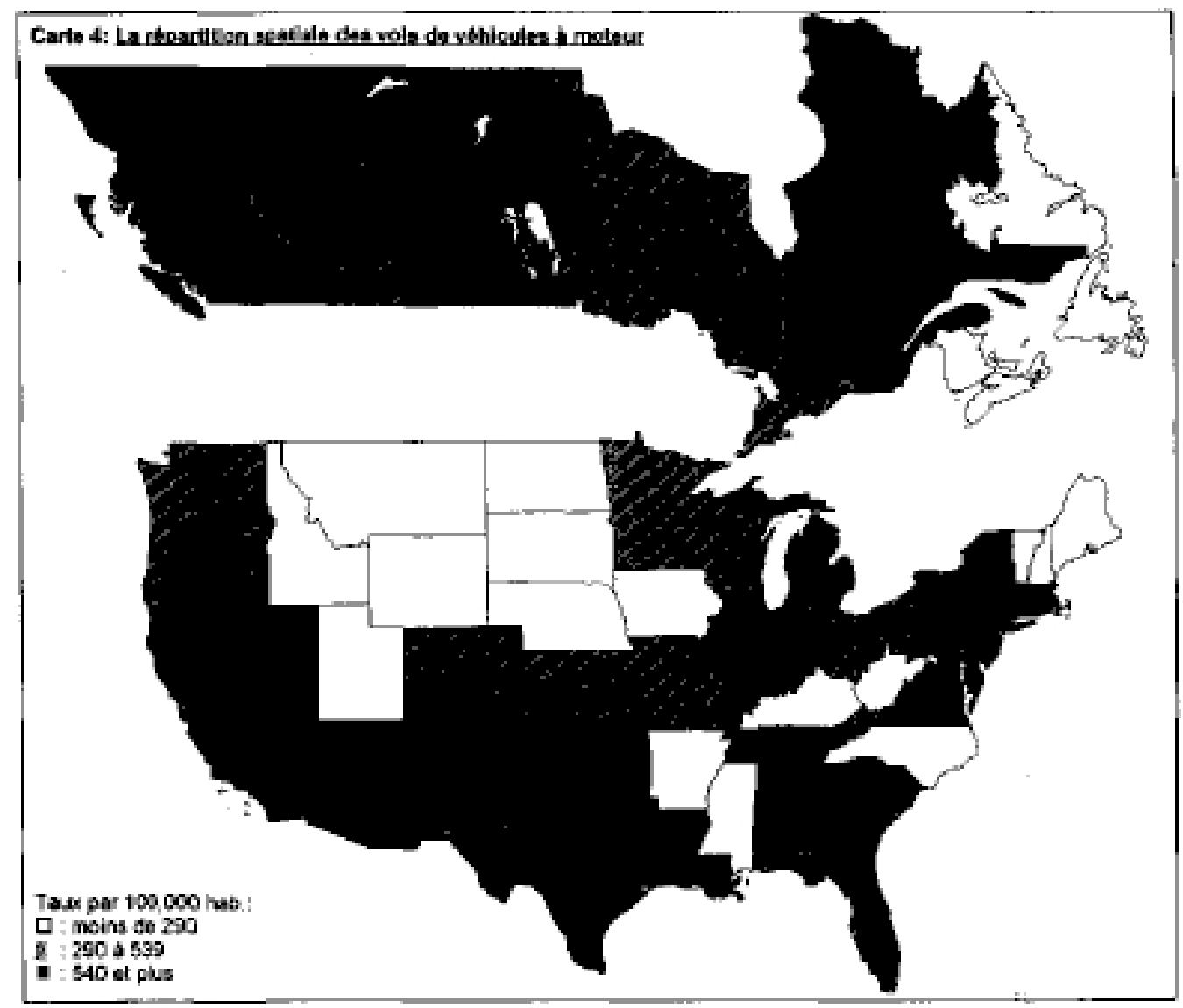

Les cartes thématiques 2, 3 et 4 illustrent la distribution spatiale des trois autres crimes étudiés. Pour le vol qualifié, le vol par effraction et le vol de véhicules à moteur, les états du Sud et du SudOuest américain ont des taux de criminalité plus élevés que ceux de la moyenne américaine. De plus, bon nombre de provinces canadiennes ne se distinguent pas des États-Unis. Le Québec est l'un des endroits où le vol qualifié est des plus fréquent, en particulier dans le cas des vols de banque (Gabor et al, 1987). L'impression générale qui se dégage d'une inspection visuelle de la distribution spatiale des trois conduites de vol est que les états du Mid-West américain seraient pris 
dans un étau constitué par le Canada et le sud des États-Unis. Cette observation est de nature à mettre en doute le caractère distinct des ÉtatsUnis et du Canada quant à la prévalence de la criminalité.

De manière à mieux cibler les explications des différences canadoaméricaines en matière de prévalence de la criminalité, il est utile d'indiquer les territoires qui se distinguent nettement des autres au niveau des quatre types de crimes étudiés. Les territoires classifiés à quatre reprises dans la catégorie signalant une forte prévalence de criminalité sont :

- l'Illinois

- la Louisiane

- la Floride

- le Texas

- le District of Columbia

- le Nevada

- la Georgie

- la Californie

Mis à part l'état du Nevada qui apparaît comme une exception en vertu de sa petite population et de son énorme industrie touristique, ces territoires, tous américains, ont plusieurs caractéristiques en commun. Ces états, où la prévalence de l'homicide et du vol est grande, possèdent tous un grand centre urbain réputé pour sa violence. Ce sont les villes de Chicago, Washington, Miami, Atlanta, la Nouvelle-Orléans, Houston et Los Angeles. Par ailleurs, cinq des sept états font partie du Sud ou du SudOuest des ÉtatsUnis, et les villes de Washington et de Chicago furent des lieux d'émigration privilégiés pour les travailleurs du coton des états du Sud durant les années quarante (Whitman, 1991). Ces données montrent donc que la criminalité aux ÉtatsUnis n'est pas distribuée aléatoirement sur l'ensemble du territoire américain, mais qu'elle est particulièrement présente dans les états du Sud et dans ceux influencés par une migration intra-nationale de populations provenant du Sud, de même que dans les états contenant une métropole réputée pour sa violence. 
À l'autre extrême du continuum se trouvent les états ou les provinces classés dans la catégorie à faible prévalence pour les quatre types de crimes étudiés. Ces territoires sont :

- Terre-Neuve

- Le Nebraska

- l'île du Prince Édouard

- Le South Dakota

- le Maine

- Le North Dakota

- le New Hampshire

- l'Idaho

- l'Iowa

- L'Utah

Ces provinces et ces états présentent aussi plusieurs caractéristiques communes. En premier lieu, aucun d'entre eux n'a de métropole. Ensuite, les habitants de ces territoires vivent surtout de la pêche ou de l'agriculture. Cependant, la vocation économique de la région ne peut être le seul déterminant de la criminalité, puisque certains états du sud des ÉtatsUnis, de même que certaines provinces des Prairies, se caractérisent aussi par une forte orientation agraire. Finalement, ces états et ces provinces sont tous situés dans la partie nord du continent.

\section{CONCLUSION}

$\underline{\text { Retour à la table des matières }}$

Il convient de rappeler que cette étude est exploratoire et que les résultats obtenus ne sont que provisoires. Les données comparatives disponibles, soit les données policières, ne constituent qu'un indicateur imparfait de l'état de la criminalité. À l'heure actuelle, les données issues des sondages canadiens de victimisation ne peuvent être comparées à celles des sondages américains, car trop de problèmes méthodologiques demeurent encore à surmonter pour les rendre comparables (Branthingham et Brantingham, 1984). Des études pilotes de délinquance révélée, comme celle de Linden et Fillmore (1981) qui compa- 
rent la délinquance des adolescents conventionnels de Richmond (Californie) et d'Edmonton, devraient être répétées sur une plus grande échelle.

Les nombreux résultats présentés dans cette étude exploratoire tendent à démontrer que les ÉtatsUnis et le Canada ne se distinguent pas nettement l'un de l'autre. Les différences agrégées observables pour l'ensemble des deux ne résistent pas à une analyse rigoureuse qui tienne compte des variations régionales intranationales des deux pays. Le cas de l'homicide est l'exception à ce constat. En effet, l'homicide est plus fréquent aux États-Unis qu'au Canada, et cela, même lorsque les ÉtatsUnis du Nord sont comparés au Canada. Il est permis de penser que la plus grande disponibilité des armes à feu aux ÉtatsUnis expliquerait pourquoi l'homicide y est plus fréquent qu'au Canada, tout en expliquant aussi pourquoi le vol par effraction est plus fréquent au nord de la frontière qu'au sud de celleci.

Les différences observées entre les taux de criminalité du Canada et des états américains comparables étant minces et non significatives, nous en sommes réduits à retourner à l'hypothèse voulant que les probabilités de victimisation des Canadiens et Américains vivant dans des états comparables soient similaires. Ainsi, bien que les valeurs, la culture et l'orientation politique des Canadiens et des Américains puissent être différentes, ces facteurs n'auraient pas d'impact direct sur les risques de victimisation des ressortissants de chacun des pays et, par conséquent, sur les probabilités pour un individu de devenir délinquant

Pour cette raison, il appert que les différences culturelles entre Américains et Canadiens observées par Lipset (1990) et Hagan (1991) n'ont pas de rapport direct avec la criminalité. Par contre, il est possible que les valeurs et la culture des citoyens aient un impact indirect sur la criminalité, par l'entremise des choix politiques et des préférences en matière de politique sociale. Par exemple, les valeurs des Américains expliquent peutêtre pourquoi les restrictions sur l'usage des armes à feu sont risquées sur le plan politique, ou pourquoi les dirigeants municipaux ont favorisé des politiques de ségrégation résidentielle de la population noire et pauvre dans certaines grandes villes. 
De manière générale, les plus grandes différences de criminalité observables entre le Canada et les ÉtatsUnis le sont dans le cadre d'une comparaison du taux de criminalité dans les grandes villes (Mundt. 1990). Ainsi, il faudrait expliquer pourquoi les grandes villes américaines sont si violentes alors que certaines métropoles canadiennes sont relativement sécuritaires. Répondre à cette question permettrait de révéler les principaux facteurs explicatifs des différences de criminalité entre le Canada et les ÉtatsUnis. Il y a fort à parier que la création de ghettos dans les grandes villes américaines a eu un impact criminogène très important. C'est donc du côté des politiques d'urbanisme et de gestion du territoire urbain qu'il faut chercher les différences réelles entre le Canada et les ÉtatsUnis.

\section{BIBLIOGRAPHIE}

Retour à la table des matières

BLUMSTEIN, A., J. COHEN, J.A. ROTH et C.A. VISHER (1986), Criminal Careers and Career Criminals, vol. 1, Washington, D.C., National Academy Press.

BRANTINGHAM, P. et P. BRANTINGHAM (1984), Patterns in crime, New York, MacMillan Publishing Cie.

CLARK, S.D. (1976), Canadian Society in Historical Perspective, Toronto, McGraw Hill.

CUSSON, M. (1990), Croissance et décroissance du crime, Paris, Presses Universitaires de France. [Texte disponible dans Les Classiques des sciences sociales. JMT.]

CUSSON Maurice, (inédit), Le meurtre aux États-Unis et au $\mathrm{Ca}$ nada : essai de criminologie comparée, École de criminologie, Université de Montréal. 
ÉLIE, D. et M. CUSSON (1992), "Autour de " Croissance et décroissance du crime »", Criminologie, vol. 25, n 1, pp. 135-147.

GABOR, T., M. BARIL, M. CUSSON, D. ELIE, M. LE BLANC et A. NORMANDEAU (1987), Armed Robbery : Cops. Robbers and Victims. Springfield, Il, Charles Thomas Publisher.

GASTIL R. D. (1971), Homicide and A Regional Culture of Violence, American Sociological Review, vol. 36, pp. 412-427.

GARTNER. R. (1990), The Victims of Homicide : A temporal and crossnational comparison, American Sociological Review, vol. 55, $\mathrm{n}^{\circ}$ 1, pp. 92-106.

HAGAN, J. (1991), The Disreputable Pleasures : Crime and Deviance in Canada, Toronto, McGrawHill.

HINDELANG, M., T. HIRSCHI et J. WEIS (1981), Measuring Delinquency, Beverly Hills, CA. Sage.

HOROWITZ, 1.L. (1973), The hemispheric connection : A critique and corrective to the entrepreneurial thesis of development with special emphasis on the Canadian case, Queen's Quarterly, vol. 80, pp. 327-359.

KENNEDY, L., R. SILVERMAN et D. FORDE (1988), Homicide from East to West : A test of the impact of culture and economic inequality on regional trends of violent crime in Canada, rapport de recherche 17, Centre for Criminological Research, University of Alberta.

KILLIAS, M. (1991), Précis de criminologie. Berne, Suisse, Éditions Staempfil \& Cie.

KLECK, G. (1979), Guns, Homicide, and Gun Control : Some assumptions and some evidence, présentation an congrès de la Midwest Sociological Society. Minneapolis. 
LENTON, R. (1989), Homicide in Canada and in the U.S.A. : A critique of the Hagan thesis. Cahiers canadiens de sociologie, vol. 14, $\mathrm{n}^{\circ} 2$, pp. 163-178.

LIPSET S.M. (1990), Continental Divide, New York, Routledge.

LINDEN, R. et C. FILLMORE (1981). A comparative study of delinquency involvement, Canadian Review of Sociology and Anthropology, vol. 18, n³, pp. 343-361.

LIZO1TE, A.J. et DJ. BORDUA (1980), Firearms ownership for sport and protection : Two divergent models, American Sociological review, vol. $45, \mathrm{n}^{\circ} 2$, pp. 229-244.

MUNDT, R. (1990), "Gun control and rates of firearms violence in Canada and the United States s, Revue Canadienne de Criminologie, vol. 32, n 1 , pp. 137-154.

U.S. DEPARTMENT OF JUSTICE (1991), Saurcebook of criminal justice Statistics, Washington, D.C., U.S. Government Printing Office.

SILVERMAN, R. et L. KENNEDY (1993), Deadly Deeds : Murder in Canada, Scarborough, Ontario, Nelson Canada.

SLOAN, JH., A.L. KELLERMAN, D.J.. REAY, J.A. FENNIS, T. KOESPELL,

F.P. RIVARA, C. RICE, L. GRAY et J. LOGERFO (1988), Handgun regulations, crime, assaults, and homicide : A tale of two cities, New England Journal of Medicine, no 319. pp. 1256-1262.

STATISTIQUE CANADA (1991), Statistique de la criminalité au Canada, Catalogue 85205, Ottawa, Centre canadien de la statistique juridique.

TRUMAN, T. (1971), A critique of Seymour Martin Lipset's article, Canadian Journal of Political Science, vol. 4, pp. 473-496. 
WRIGHT, J.D., PH. ROSSI et K. DALY (1982), Under the Gun : Weapons, crime and violence in America. New York, Aldine de Gruyler.

WHITMAN, D. (L991), " The migrants' tale and ghetto culture », The Public Interest, $\mathrm{n}^{\circ} 105$ (automne).

Fin du texte 